\title{
Biomimetic Material-Assisted Delivery of Human Embryonic Stem Cell Derivatives for Enhanced In Vivo Survival and Engraftment
}

\author{
Harsha Kabra, ${ }^{\dagger, \dagger}$ Yongsung Hwang, ${ }^{\dagger \dagger}$ Han Liang Lim, ${ }^{\dagger}$ Mrityunjoy Kar, ${ }^{\dagger}$ Gaurav Arya, ${ }^{\S}$
} and Shyni Varghese*, $\dagger$ Department of Bioengineering and ${ }^{\S}$ Department of NanoEngineering, University of California, San Diego, La Jolla, California 92093 ,
United States

Supporting Information

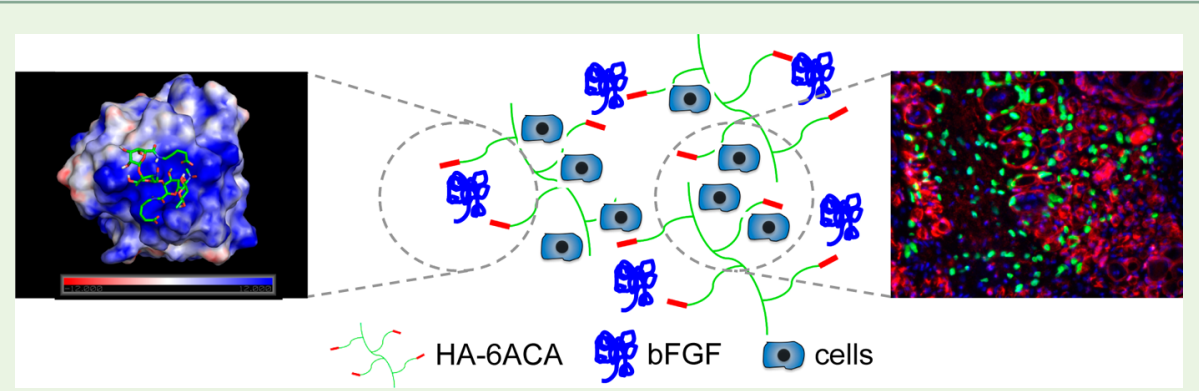

ABSTRACT: The ability of human embryonic stem cells (hESCs) and their derivatives to differentiate and contribute to tissue repair has enormous potential to treat various debilitating diseases. However, improving the in vivo viability and function of the transplanted cells, a key determinant of translating cell-based therapies to the clinic, remains a daunting task. Here, we develop a hybrid biomaterial consisting of hyaluronic acid (HA) grafted with 6-aminocaproic acid moieties (HA-6ACA) to improve cell delivery and their subsequent in vivo function using skeletal muscle as a model system. Our findings show that the biomimetic material-assisted delivery of hESC-derived myogenic progenitor cells into cardiotoxin-injured skeletal muscles of NOD/SCID mice significantly promotes survival and engraftment of transplanted cells in a dose-dependent manner. The donor cells were found to contribute to the regeneration of damaged muscle fibers and to the satellite cell (muscle specific stem cells) compartment. Such biomimetic cell delivery vehicles that are cost-effective and easy-to-synthesize could play a key role in improving the outcomes of other stem cell-based therapies.

KEYWORDS: human embryonic stem cells, myogenesis, biomaterials, hyaluronic acid, stem cell transplantation

\begin{abstract}
A lthough transplantation of stem cells has been touted as a promising strategy for treating impaired skeletal muscles, the therapeutic potential of such an approach has been hampered by poor to modest survival, low retention, and lack of integration of the transplanted cells with the host tissue. ${ }^{1-4}$ Thus, there is a strong interest in developing delivery strategies that can improve the survival, continued differentiation, and contribution of the transplanted cells to muscle tissue repair. ${ }^{5}$ Here, we describe the development of a hybrid biomaterial containing hyaluronic acid (HA) grafted with 6-aminocaproic acid molecules (6ACA), hereafter termed as HA-6ACA, and demonstrate that HA-6ACA-assisted administration of hESCderived cells significantly improves the survival and engraftment of transplanted cells within an injured skeletal muscle tissue.

HA, a nonsulfated linear glycosaminoglycan, is a well-studied biomaterial for cell and drug delivery, soft-tissue repair, and tissue engineering. ${ }^{6-14} \mathrm{HA}$ is a key extracellular matrix molecule found in the interstitial matrix of skeletal muscle, and has been implicated in mediating interactions with various proteins and growth factors, cell migration, cell signaling, matrix reorganization, and regeneration. ${ }^{15-21} \mathrm{HA}$ also interacts with cells through the CD44 receptor, which is expressed in most
\end{abstract}

cells. $^{22-25}$ Several studies have suggested that HA does not exhibit strong binding to basic fibroblast growth factor (bFGF); ${ }^{26,27}$ but can weakly interact with bFGF through positively charged regions. We hypothesize that biomaterials that can regulate bFGF signaling could have an added advantage as cell delivery vehicles given that bFGF signaling plays a key role in skeletal muscle tissue homeostasis and function by maintaining a balance between proliferation and differentiation of myogenic progenitor cells. ${ }^{28,29}$ Hence, we endowed the HA molecules with 6ACA moieties to improve their interactions with bFGF. Previously, we have shown that incorporating 6ACA moieties onto hydrogels can impart them with unique features such as healing, ${ }^{30}$ biomineralization, ${ }^{31}$ and increased protein adsorption and cell-matrix interaction. ${ }^{32}$

The synthesis scheme of HA-6ACA is shown in Figure 1. Subsequent characterization using ${ }^{1} \mathrm{H}$ NMR and FTIR spectra (see Figure S1A, B in the Supporting Information) showed successful grafting ( $60-70 \%)$ of 6 ACA moieties onto the HA

Received: September 22, 2014

Accepted: November 21, 2014

Published: November 21, 2014 


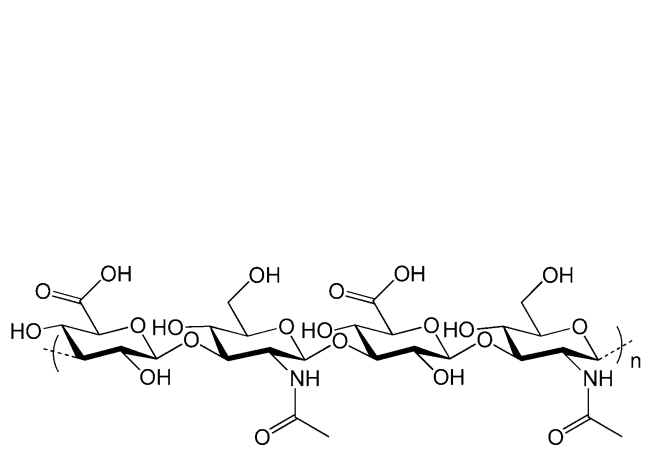

Hyaluronic acid (HA)

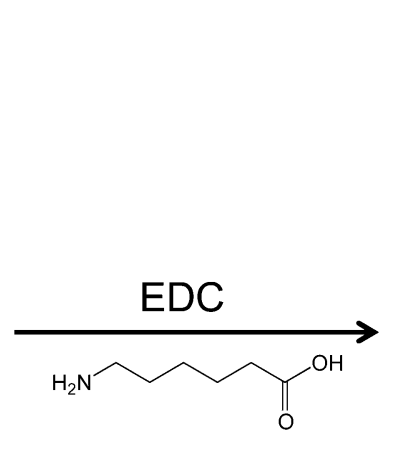

6-aminocaproic acid

(6ACA)

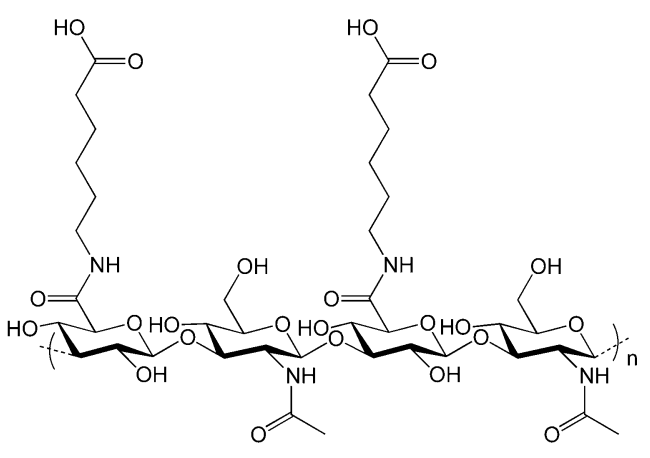

HA-6ACA

Figure 1. Reaction scheme for the synthesis of HA-6ACA.
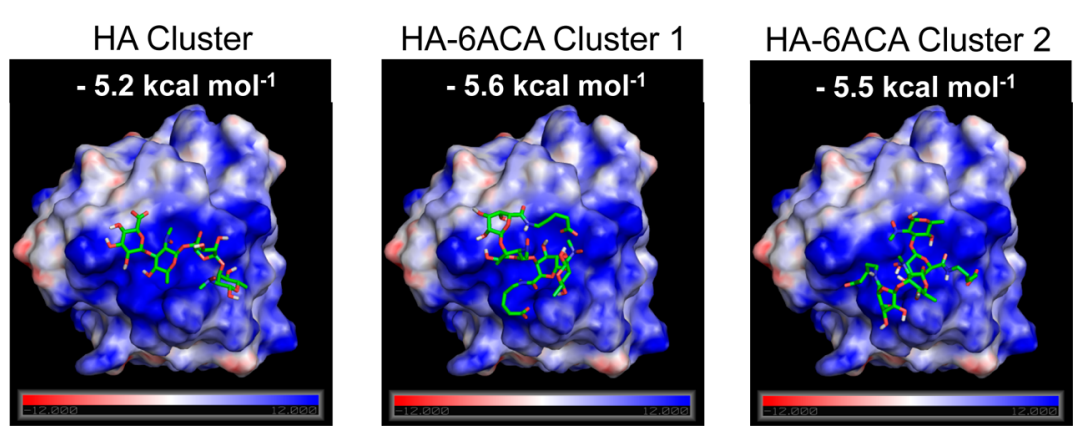

Figure 2. Lowest-energy configuration from the most populated binding cluster(s) of HA and HA-6ACA. This represents the most likely configuration assumed by the ligand when interacting with bFGF. The computed binding energies for the molecules are also provided.

backbone. To investigate the effect of 6ACA incorporation on the ability of HA to interact with bFGF, we carried out molecular docking studies and enzyme-linked immunosorbent assay (ELISA) measurements. Our docking calculations yielded hundreds of low-energy configurations of bFGF-bound HA6ACA and HA that were further categorized into clusters of closely resembling configurations (Figure S2A). The lowest energy configuration in the most populated cluster is generally considered as the putative binding mode, and its corresponding energy value the binding free energy. ${ }^{33}$ Because HA-6ACA exhibits two highly populated clusters, both binding modes are considered equally likely. Hence, HA-6ACA, which exhibits binding free energies of $-5.6 \mathrm{kcal} \mathrm{mol}^{-1}$ or $-5.5 \mathrm{kcal} \mathrm{mol}^{-1}$ for the two modes, binds more strongly to bFGF than HA, which exhibits a binding free energy of $-5.2 \mathrm{kcal} \mathrm{mol}^{-1}$ (Figure 2). To probe the molecular basis for the observed higher affinity of HA-6ACA to bFGF, we compared the lowest-energy bFGFbound configuration of the HA-6ACA and HA molecules. We find that the terminal carboxyl group of dangling side chain of 6ACA acts as a flexible arm that wraps around the positively charged region of bFGF, allowing it to interact easily with positively charged amino acids on the surface of bFGF. The fact that HA-6ACA exhibits multiple binding modes suggests a high degree of flexibility in how HA-6ACA can bind to bFGF, making its binding even more favorable from an entropic perspective.

These results from our computational analyses were further verified by ELISA. In order to immobilize the HA-6ACA molecules for the ELISA measurements, we used semiinterpenetrating networks (semi-iPNs). The semi-IPNs were created by incorporating the linear HA or HA-6ACA molecules into chemically cross-linked polyethylene glycol diacrylate
(PEGDA) hydrogels, which are known to be antifouling. ${ }^{34}$ We use PEGDA with a molecular weight of $508 \mathrm{Da}$ to ensure a tight network with minimal swelling in an aqueous milieu. The amounts of adsorbed bFGF onto both semi-iPN HA-PEGDA and semi-iPN HA-6ACA-PEGDA were also compared against those on PEGDA hydrogels. As shown in Figure S3A, the amount of bFGF adsorbed by semi-iPN containing HA-6ACA molecules was significantly higher than that on corresponding semi-iPN containing HA molecules, corroborating our computational findings. No significant differences in bFGF measurements were found between semi-iPN HA-PEGDA and PEGDA networks. The positive measurements observed here could be attributed to the absorption of bFGF solution into the polymer network. ${ }^{35}$ The same semi-iPN experimental setup was used to determine the interaction of HA and HA-6ACA molecules with various ECM proteins relevant to skeletal muscle tissues-collagen type I, collagen type IV, and laminin. The results in Figure S3B-D show higher levels of interactions between the ECM proteins and HA-6ACA-PEGDA semi-iPN hydrogels compared to its HA-PEGDA and PEGDA counterparts. This is in accordance with our previous report that demonstrates the ability of 6ACA to promote nonspecific adsorption of proteins from their extracellular milieu. ${ }^{32}$

Hyaluronan molecules exhibit rapid turnover in vivo and chemical modifications of HA have been used to retard their degradation kinetics. $^{36}$ To determine the effect of 6ACA incorporation on the degradation of $\mathrm{HA}$, we used a modified uronic acid assay, which shows approximately $45 \%$ less degradation of HA-6ACA compared to $\mathrm{HA}$ in the presence of hyaluronidase, whereas no detectable degradation products were observed in the controls-HA and HA-6ACA incubated in PBS without hyaluronidase (Figure S4). The slower 

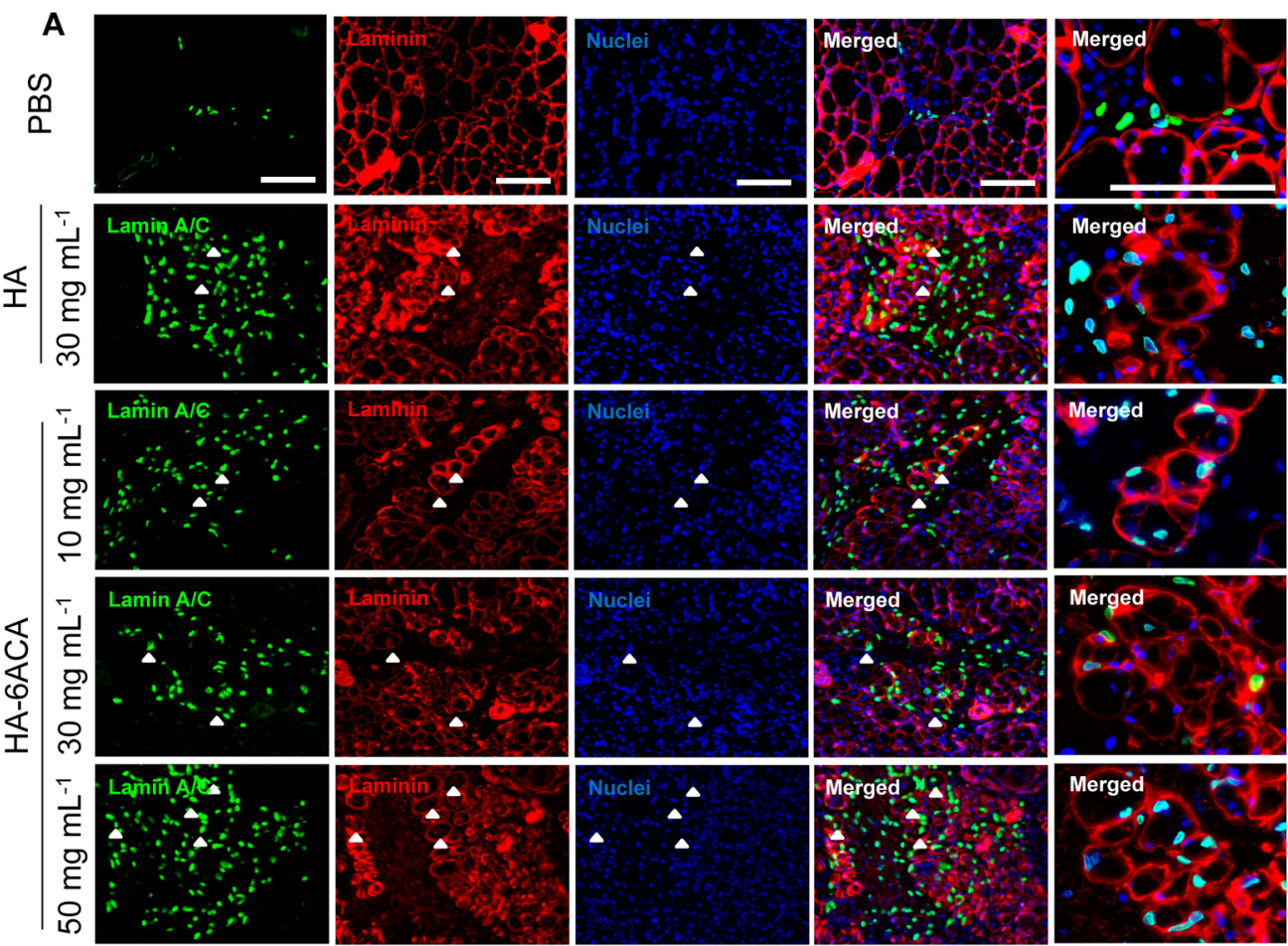

B
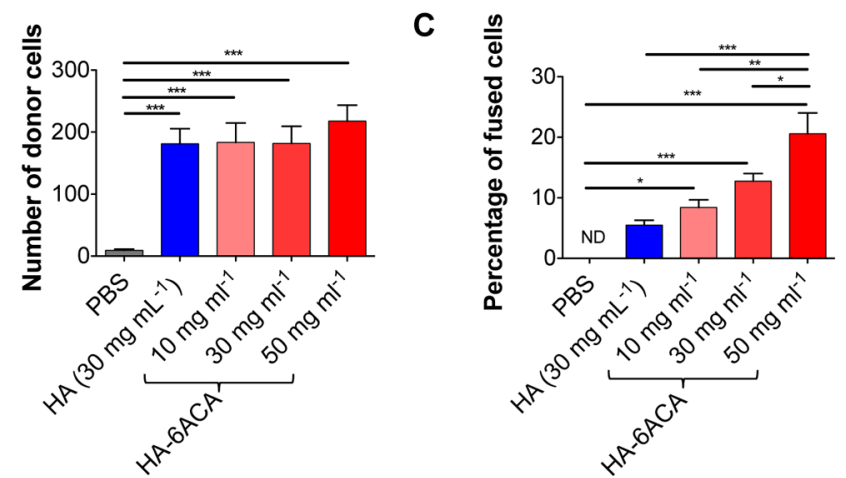

Figure 3. In vivo survival and engraftment of transplanted hESC-derived myogenic progenitor cells. (A) Immunofluorescence staining for humanspecific lamin A/C (green), laminin (red), and nuclei (blue). Scale bar $=100 \mu \mathrm{m}$. Quantitative estimation of (B) the total donor cells and (C) the contribution of donor cells to the host myofibers 14 days post-transplantation.

degradation of HA-6ACA suggests that the terminal carboxyl groups of the pendant side chains hinder the accessibility of hyaluronidase enzymes to their binding sites on HA backbone. The slow degradation of HA-6ACA along with their ability to interact with growth factors and ECM proteins could have multiple effects such as modulating signaling and prolonging the half-life of growth factors by inhibiting their proteolytic cleavage. $^{32}$

We next determined the effect of HA-6ACA in promoting in vivo survival and function of transplanted hESC-derived progenitor cells. Previously, we have shown that hESC-derived PDGFRA $^{+}$cells can undergo myogenic differentiation in vitro and in vivo. ${ }^{37,38}$ However, when implanted in vivo, only a few cells were found to be viable post-transplantation with a majority of the survived donor cells residing within the interstitial space. ${ }^{37}$ To assess the potential of HA-6ACA to support the survival and function of transplanted cells, hESCderived myogenically committed cells were injected using HA6ACA molecules of varying concentrations $(10,30$, and $50 \mathrm{mg}$ $\mathrm{mL}^{-1}$ ) into cardiotoxin injured tibialis anterior (TA) muscles of NOD/SCID mice. The HA-6ACA-assisted survival and engraftment of donor cells 14 days post-transplantation were compared against the same cell population injected using saline solution or $30 \mathrm{mg} \mathrm{mL}^{-1} \mathrm{HA}$.

The TA muscles were harvested and characterized to evaluate the survival and in vivo engraftment efficiency of the transplanted donor cells. The muscle sections were stained for human-specific lamin A/C, laminin, and nuclei (Figure 3A). The positive staining for lamin $\mathrm{A} / \mathrm{C}$ indicates the presence of transplanted hESC-derived cells. Interestingly, the use of HA6ACA dramatically improved the survival and engraftment of transplanted donor cells. Quantification of histological sections showed a striking $\sim 200$ fold increase in donor cells within the host tissue when they were transplanted using either HA or HA-6ACA compared to that of saline groups (Figure 3B). The HA-6ACA-assisted in vivo survival of transplanted donor cells was found to be independent of their concentration. A similar increase in cell survival was also observed in the presence of 30 $\mathrm{mg} \mathrm{mL} \mathrm{m}^{-1} \mathrm{HA}$. In addition to supporting viability of transplanted cells, both HA-6ACA and HA also promoted the dissemination of transplanted cells within the host tissue. In contrast to HA-6ACA and HA, only a few donor cells were 

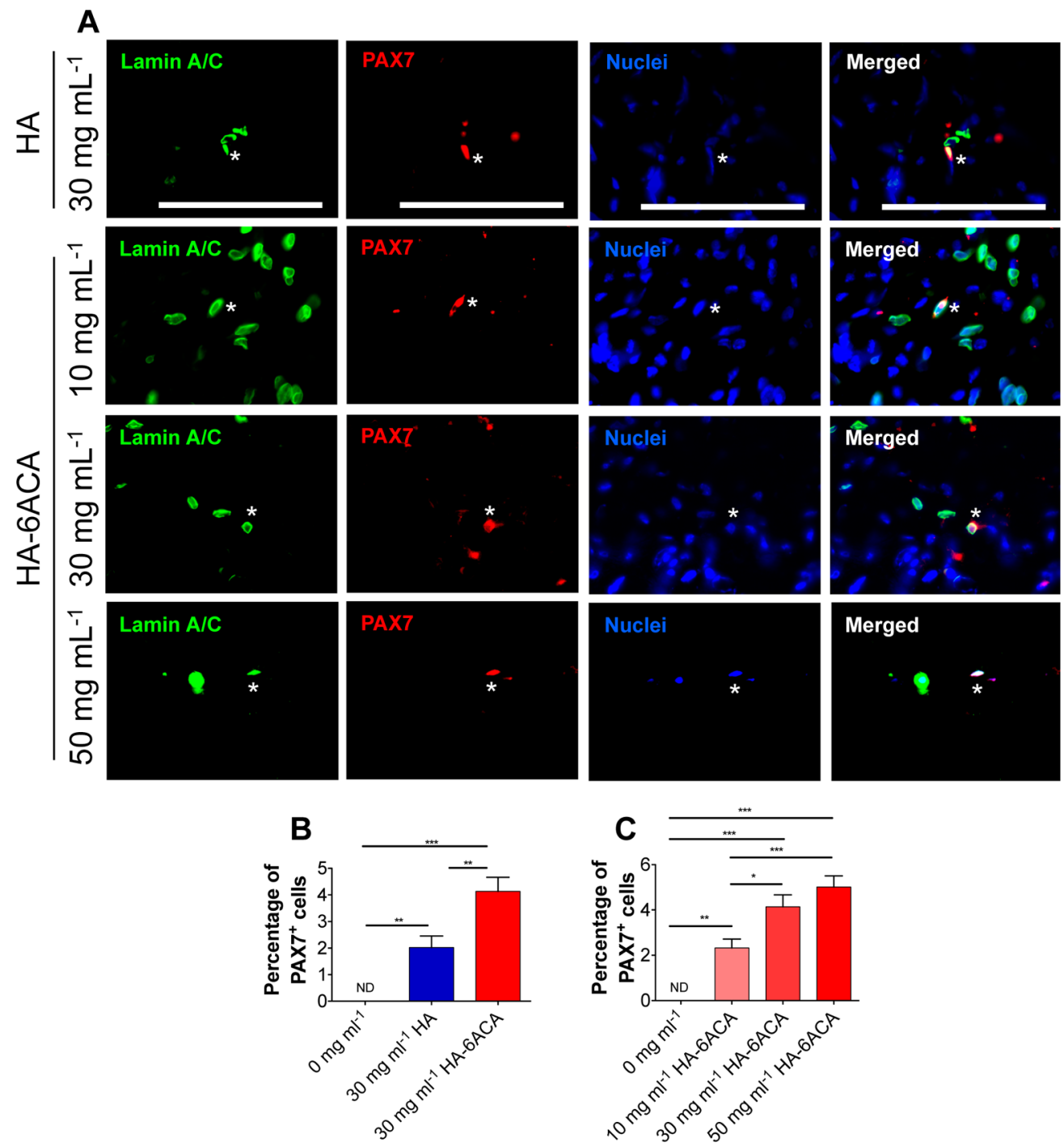

Figure 4. Biomimetic material-assisted contribution of donor cells to the satellite cell compartment: (A) Immunofluorescence staining for humanspecific lamin A/C (green), PAX7 (red) for satellite cells, and nuclei (blue). Scale bar $=100 \mu \mathrm{m}$. (B, C) Quantification of the donor cell-derived satellite cells.

found to be viable when saline was used as a delivery vehicle and the majority of the donor cells were found within the interstitial space nearby the host muscle fibers akin to previous reports. $^{37}$

Although similar levels of cell viability were observed between HA and HA-6ACA, HA-6ACA molecules had a significant effect in determining the contribution of donor cells toward regeneration of damaged muscles. Histological analyses of donor cell contribution to the regenerating myofibers showed a remarkably higher number of fused donor cells when the cells were injected with $50 \mathrm{mg} \mathrm{mL}^{-1} \mathrm{HA}-6 \mathrm{ACA}$ (Figure 3A, $\mathrm{C}$ and Figure S5). The contribution of donor cells to tissue repair was found to be dependent on the concentration of HA6ACA (Figure 3C). When the cells were injected with $30 \mathrm{mg}$ $\mathrm{mL}^{-1} \mathrm{HA}$, only few cells were found to integrate with the host muscle fibers, whereas in the case of saline assisted cell transplantation no donor cells were fused with the host muscle fibers. Even though cell transplantation using $30 \mathrm{mg} \mathrm{mL}^{-1} \mathrm{HA}$ exhibited some contribution of the donor cells toward the host tissue, the total number of cells integrated with the host myofibers was significantly lower than that observed when the cells were delivered using $10 \mathrm{mg} \mathrm{mL} \mathrm{mL}^{-1} \mathrm{HA}-6 \mathrm{ACA}$, the lowest concentration of HA-6ACA used.

We also investigated the donor cell contribution to the satellite cell compartment by staining serial muscle sections with human-specific lamin A/C, PAX7 (a satellite cell marker), and nuclei. Our results show that although only a small percentage of the transplanted cells contributed to satellite cell compartment, the donor cell-derived satellite cells were only found in cells administered using either HA or HA-6ACA (Figure 4). Similar to the contribution of donor cells to the host myofibers, the donor cell-derived satellite cells exhibited a monotonic increase with HA-6ACA concentration. The cells administered using $30 \mathrm{mg} \mathrm{mL}^{-1}$ of $\mathrm{HA}$ had the lowest number of donor cell-derived satellite cells, whereas cells injected using $50 \mathrm{mg} \mathrm{mL}^{-1} \mathrm{HA}-6 \mathrm{ACA}$ showed the highest contribution to the satellite cell compartment.

Our results clearly demonstrate the beneficial effect of HA6ACA in promoting the survival and engraftment of transplanted hESC-derived myogenic progenitor cells. The observed beneficial effect of HA-based biomaterials (HA and HA-6ACA) on improving the survival of transplanted cells could be attributed to various biological functions of HA. HA molecules 
have been shown to interact with cells through cell surface hyaluronic acid receptor, CD $44 .^{39,40}$ As reported previously, a majority of HUES9 derived mesoderm progenitor cells are positive for CD44 cell surface markers. ${ }^{23,24,41-43}$ So we anticipate that the biological functions of HA molecules along with their plausible interactions with the cells through CD44 could be contributing to the enhanced cell survival. Among HA and HA-6ACA, the latter was found to have a better effect on the contribution of donor cells to the myofibers and satellite cell pool. We speculate that this could possibly be due to enhanced interaction between HA-6ACA and growth factors such as bFGF. The enhanced interaction of HA-6ACA molecules with bFGF along with its slower degradation could facilitate sequestration of bFGF away from cell surface and subsequent modulation of bFGF signaling. Previous studies have shown that continuous and high levels of bFGF in cultures inhibit myogenic differentiation of progenitor cells while suppressing bFGF signaling (or low levels of bFGF) promotes in vitro myogenesis of myoblasts. ${ }^{28,29}$ In native tissue, bFGF is regulated by heparin and heparan sulfate proteoglycans, ${ }^{26}$ and previously we have shown that synthetic analogues of heparin in culture conditions can promote myogenic differentiation of C2C12 cells to myotubes through bFGF signaling. ${ }^{44}$

In summary, this study demonstrates that HA-6ACA-assisted delivery of hESC-derived myogenic progenitor cells significantly improves the in vivo survival, differentiation, and integration of transplanted donor cells to the host tissues. Such biomimetic material-based cell delivery vehicles that are cost-effective and easy-to-synthesize might be able to augment the therapeutic outcomes of stem cell-based therapy and accelerate their use from the bench to the bedside.

\section{ASSOCIATED CONTENT}

\section{S Supporting Information}

The following file is available free of charge on the ACS Publications website at DOI: 10.1021/ab500021a:

Experimental details and characterization data ( $\underline{\mathrm{PDF}})$

\section{AUTHOR INFORMATION}

\section{Corresponding Author}

*E-mail: svarghese@ucsd.edu.

\section{Author Contributions}

${ }^{\ddagger}$ H.K. and Y.H contributed equally to this work. H.K., Y.H., and S.V. conceived the idea and designed research. H.K and Y.H performed cell and animal experiments, H.L. and G.A. performed the computational analysis, M.K. synthesized and characterized the biomaterial. H.K., Y.H., H.L., M.K., G.A., and S.V analyzed and interpreted the data and wrote manuscript.

\section{Funding}

This work was funded by the California Institute of Regenerative Medicine (RN2-00945).

\section{Notes}

The authors declare no competing financial interest.

\section{ACKNOWLEDGMENTS}

The authors acknowledge Karl E. Marquez for experimental assistance with FACS. We also acknowledge the Developmental Studies Hybridoma Bank for providing the PAX7 antibody generated by Kawakami Lab. The antibodies are developed under the auspices of the NICHD and maintained by The
University of Iowa, Department of Biology, Iowa City, IA 52242.

\section{REFERENCES}

(1) Qu, Z.; Balkir, L.; van Deutekom, J. C.; Robbins, P. D.; Pruchnic, R; Huard, J. Development of approaches to improve cell survival in myoblast transfer therapy. J. Cell Biol. 1998, 142 (5), 1257-67.

(2) Barberi, T.; Bradbury, M.; Dincer, Z.; Panagiotakos, G.; Socci, N. D.; Studer, L. Derivation of engraftable skeletal myoblasts from human embryonic stem cells. Nat. Med. 2007, 13 (5), 642-8.

(3) Darabi, R.; Gehlbach, K.; Bachoo, R. M.; Kamath, S.; Osawa, M.; Kamm, K. E.; Kyba, M.; Perlingeiro, R. C. Functional skeletal muscle regeneration from differentiating embryonic stem cells. Nat. Med. 2008, 14 (2), 134-43.

(4) Goudenege, S.; Lebel, C.; Huot, N. B.; Dufour, C.; Fujii, I.; Gekas, J.; Rousseau, J.; Tremblay, J. P. Myoblasts derived from normal hESCs and dystrophic hiPSCs efficiently fuse with existing muscle fibers following transplantation. Mol. Ther. 2012, 20 (11), 2153-67.

(5) Bentzinger, C. F.; Wang, Y. X.; von Maltzahn, J.; Rudnicki, M. A. The emerging biology of muscle stem cells: implications for cell-based therapies. BioEssays 2013, 35 (3), 231-41.

(6) Solchaga, L. A.; Gao, J.; Dennis, J. E.; Awadallah, A.; Lundberg, M.; Caplan, A. I.; Goldberg, V. M. Treatment of osteochondral defects with autologous bone marrow in a hyaluronan-based delivery vehicle. Tissue Eng. 2002, 8 (2), 333-47.

(7) Rossi, C. A.; Flaibani, M.; Blaauw, B.; Pozzobon, M.; Figallo, E.; Reggiani, C.; Vitiello, L.; Elvassore, N.; De Coppi, P. In vivo tissue engineering of functional skeletal muscle by freshly isolated satellite cells embedded in a photopolymerizable hydrogel. FASEB J. 2011, 25 (7), 2296-304.

(8) Burdick, J. A.; Prestwich, G. D. Hyaluronic acid hydrogels for biomedical applications. Adv. Mater. 2011, 23 (12), H41-56.

(9) Gerecht, S.; Burdick, J. A.; Ferreira, L. S.; Townsend, S. A.; Langer, R.; Vunjak-Novakovic, G. Hyaluronic acid hydrogel for controlled self-renewal and differentiation of human embryonic stem cells. Proc. Natl. Acad. Sci. U.S.A. 2007, 104 (27), 11298-303.

(10) Shu, X. Z.; Ahmad, S.; Liu, Y.; Prestwich, G. D. Synthesis and evaluation of injectable, in situ crosslinkable synthetic extracellular matrices for tissue engineering. J. Biomed. Mater. Res., Part A 2006, 79 (4), 902-12.

(11) Chung, C.; Burdick, J. A. Influence of three-dimensional hyaluronic acid microenvironments on mesenchymal stem cell chondrogenesis. Tissue Eng., Part A 2009, 15 (2), 243-54.

(12) Luo, Y.; Bernshaw, N. J.; Lu, Z. R.; Kopecek, J.; Prestwich, G. D. Targeted delivery of doxorubicin by HPMA copolymer-hyaluronan bioconjugates. Pharm. Res. 2002, 19 (4), 396-402.

(13) Luo, Y.; Prestwich, G. D. Synthesis and selective cytotoxicity of a hyaluronic acid-antitumor bioconjugate. Bioconjugate Chem. 1999, 10 (5), 755-63.

(14) Luo, Y.; Ziebell, M. R.; Prestwich, G. D. A hyaluronic acid-taxol antitumor bioconjugate targeted to cancer cells. Biomacromolecules 2000, 1 (2), 208-18.

(15) Cheung, W. F.; Cruz, T. F.; Turley, E. A. Receptor for hyaluronan-mediated motility (RHAMM), a hyaladherin that regulates cell responses to growth factors. Biochem. Soc. Trans. 1999, 27 (2), 135-141 DOI: $10.1042 /$ bst0270135.

(16) Tammi, R. H.; Passi, A. G.; Rilla, K.; Karousou, E.; Vigetti, D.; Makkonen, K.; Tammi, M. I. Transcriptional and post-translational regulation of hyaluronan synthesis. FEBS J. 2011, 278 (9), 1419-28.

(17) Hunt, L. C.; Gorman, C.; Kintakas, C.; McCulloch, D. R.; Mackie, E. J.; White, J. D. Hyaluronan synthesis and myogenesis: a requirement for hyaluronan synthesis during myogenic differentiation independent of pericellular matrix formation. J. Biol. Chem. 2013, 288 (18), 13006-21.

(18) Calve, S.; Isaac, J.; Gumucio, J. P.; Mendias, C. L. Hyaluronic acid, HAS1, and HAS2 are significantly upregulated during muscle hypertrophy. Am. J. Physiol. Cell Physiol. 2012, 303 (5), C577-88.

(19) Toole, B. P. Hyaluronan: from extracellular glue to pericellular cue. Nat. Rev. Cancer 2004, 4 (7), 528-39. 
(20) Prestwich, G. D. Hyaluronic acid-based clinical biomaterials derived for cell and molecule delivery in regenerative medicine. $J$. Controlled Release 2011, 155 (2), 193-9.

(21) Purcell, B. P.; Elser, J. A.; Mu, A.; Margulies, K. B.; Burdick, J. A. Synergistic effects of SDF-1alpha chemokine and hyaluronic acid release from degradable hydrogels on directing bone marrow derived cell homing to the myocardium. Biomaterials 2012, 33 (31), 7849-57.

(22) Sherwood, R. I.; Christensen, J. L.; Conboy, I. M.; Conboy, M. J.; Rando, T. A.; Weissman, I. L.; Wagers, A. J. Isolation of adult mouse myogenic progenitors: functional heterogeneity of cells within and engrafting skeletal muscle. Cell 2004, 119 (4), 543-54.

(23) Darabi, R.; Arpke, R. W.; Irion, S.; Dimos, J. T.; Grskovic, M.; Kyba, M.; Perlingeiro, R. C. Human ES- and iPS-derived myogenic progenitors restore DYSTROPHIN and improve contractility upon transplantation in dystrophic mice. Cell Stem Cell 2012, 10 (5), 610-9.

(24) Meng, J.; Adkin, C. F.; Xu, S. W.; Muntoni, F.; Morgan, J. E. Contribution of human muscle-derived cells to skeletal muscle regeneration in dystrophic host mice. PLoS One 2011, 6 (3), e17454.

(25) Zhu, H.; Mitsuhashi, N.; Klein, A.; Barsky, L. W.; Weinberg, K.; Barr, M. L.; Demetriou, A.; Wu, G. D. The role of the hyaluronan receptor CD44 in mesenchymal stem cell migration in the extracellular matrix. Stem Cells 2006, 24 (4), 928-35.

(26) Bashkin, P.; Doctrow, S.; Klagsbrun, M.; Svahn, C. M.; Folkman, J.; Vlodavsky, I. Basic fibroblast growth factor binds to subendothelial extracellular matrix and is released by heparitinase and heparin-like molecules. Biochemistry 1989, 28 (4), 1737-43.

(27) Malkowski, A.; Sobolewski, K.; Jaworski, S.; Bankowski, E. FGF binding by extracellular matrix components of Wharton's jelly. Acta Biochim. Pol. 2007, 54 (2), 357-63.

(28) Clegg, C. H.; Linkhart, T. A.; Olwin, B. B.; Hauschka, S. D. Growth factor control of skeletal muscle differentiation: commitment to terminal differentiation occurs in G1 phase and is repressed by fibroblast growth factor. J. Cell Biol. 1987, 105 (2), 949-56.

(29) Allen, R. E.; Rankin, L. L. Regulation of satellite cells during skeletal muscle growth and development. Proc. Soc. Exp. Biol. Med. 1990, 194 (2), 81-6.

(30) Phadke, A.; Zhang, C.; Arman, B.; Hsu, C. C.; Mashelkar, R. A.; Lele, A. K.; Tauber, M. J.; Arya, G.; Varghese, S. Rapid self-healing hydrogels. Proc. Natl. Acad. Sci. U. S. A. 2012, 109 (12), 4383-8.

(31) Phadke, A.; Shih, Y. R.; Varghese, S. Mineralized synthetic matrices as an instructive microenvironment for osteogenic differentiation of human mesenchymal stem cells. Macromol. Biosci. 2012, 12 (8), 1022-32.

(32) Ayala, R.; Zhang, C.; Yang, D.; Hwang, Y.; Aung, A.; Shroff, S. S.; Arce, F. T.; Lal, R.; Arya, G.; Varghese, S. Engineering the cellmaterial interface for controlling stem cell adhesion, migration, and differentiation. Biomaterials 2011, 32 (15), 3700-11.

(33) Yang, D.; Arya, G. Structure and binding of the H4 histone tail and the effects of lysine 16 acetylation. Phy. Chem. Chem. Phys. 2011, 13 (7), 2911-21.

(34) Ekblad, T.; Bergstrom, G.; Ederth, T.; Conlan, S. L.; Mutton, R.; Clare, A. S.; Wang, S.; Liu, Y.; Zhao, Q.; D’Souza, F.; Donnelly, G. T.; Willemsen, P. R.; Pettitt, M. E.; Callow, M. E.; Callow, J. A.; Liedberg, B. Poly(ethylene glycol)-containing hydrogel surfaces for antifouling applications in marine and freshwater environments. Biomacromolecules 2008, 9 (10), 2775-83.

(35) Zieris, A.; Prokoph, S.; Welzel, P. B.; Grimmer, M.; Levental, K. R.; Panyanuwat, W.; Freudenberg, U.; Werner, C. Analytical approaches to uptake and release of hydrogel-associated FGF-2. J. Mater. Sci. Mater. Med. 2010, 21 (3), 915-23.

(36) Sahoo, S.; Chung, C.; Khetan, S.; Burdick, J. A. Hydrolytically degradable hyaluronic acid hydrogels with controlled temporal structures. Biomacromolecules 2008, 9 (4), 1088-92.

(37) Hwang, Y.; Suk, S.; Lin, S.; Tierney, M.; Du, B.; Seo, T.; Mitchell, A.; Sacco, A.; Varghese, S. Directed in vitro myogenesis of human embryonic stem cells and their in vivo engraftment. PLoS One 2013, 8 (8), e72023.
(38) Hwang, Y.; Suk, S.; Shih, Y. R.; Seo, T.; Du, B.; Xie, Y.; Li, Z.; Varghese, S. WNT3A promotes myogenesis of human embryonic stem cells and enhances in vivo engraftment. Sci. Rep. 2014, 4, 5916.

(39) Peach, R. J.; Hollenbaugh, D.; Stamenkovic, I.; Aruffo, A. Identification of hyaluronic acid binding sites in the extracellular domain of CD44. J. Cell Biol. 1993, 122 (1), 257-64.

(40) Ahrens, T.; Assmann, V.; Fieber, C.; Termeer, C.; Herrlich, P.; Hofmann, M.; Simon, J. C. CD44 is the principal mediator of hyaluronic-acid-induced melanoma cell proliferation. J. Invest. Dermatol. 2001, 116 (1), 93-101.

(41) Mahmood, A.; Harkness, L.; Schroder, H. D.; Abdallah, B. M.; Kassem, M. Enhanced differentiation of human embryonic stem cells to mesenchymal progenitors by inhibition of TGF-beta/activin/nodal signaling using SB-431542. J. Bone Miner. Res. 2010, 25 (6), 1216-33.

(42) de Peppo, G. M.; Svensson, S.; Lenneras, M.; Synnergren, J.; Stenberg, J.; Strehl, R; Hyllner, J.; Thomsen, P.; Karlsson, C. Human embryonic mesodermal progenitors highly resemble human mesenchymal stem cells and display high potential for tissue engineering applications. Tissue Eng., Part A 2010, 16 (7), 2161-82.

(43) Lian, Q.; Lye, E.; Suan Yeo, K.; Khia Way Tan, E.; Salto-Tellez, M.; Liu, T. M.; Palanisamy, N.; El Oakley, R. M.; Lee, E. H.; Lim, B.; Lim, S. K. Derivation of clinically compliant MSCs from CD105+, CD24- differentiated human ESCs. Stem Cells 2007, 25 (2), 425-36.

(44) Sangaj, N.; Kyriakakis, P.; Yang, D.; Chang, C. W.; Arya, G.; Varghese, S. Heparin mimicking polymer promotes myogenic differentiation of muscle progenitor cells. Biomacromolecules 2010, 11 (12), 3294-300. 